\title{
Disruption of Orbitofronto-Striatal Functional Connectivity Underlies Maladaptive Persistent Behaviors in Alcohol-Dependent Patients
}

\author{
Seojung Lee ${ }^{1}$, Eun Lee ${ }^{1}$, Jeonghun $\mathrm{Ku}^{2}$, Kang-Jun Yoon ${ }^{3}$, Kee Namkoong ${ }^{1}$ and Young-Chul Jung ${ }^{\circledR}$ \\ ${ }^{1}$ Department of Psychiatry and Institute of Behavioral Science in Medicine, Yonsei University College of Medicine, Seoul, Republic of Korea \\ 2Department of Biomedical Engineering, Keimyung University, Daegu, Republic of Korea \\ ${ }^{3}$ Department of Neurosurgery, St. Peter's Hospital, Seoul, Republic of Korea
}

Objective Alcohol dependence is characterized by persistent alcohol-seeking despite negative consequences. Previous studies suggest that maladaptive persistent behaviors reflect alcohol-induced brain changes that cause alterations in the cortico-striatal-limbic circuit.

Methods Twenty one alcohol dependent patients and 24 age-matched healthy controls performed a decision-making task during functional MRI. We defined the medial orbitofrontal cortex (mOFC) as a region-of-interest and performed seed-based functional connectivity analysis.

Results Healthy controls were more flexible in adapting an alternative behavioral strategy, which correlated with stronger mOFC-dorsal striatum functional connectivity. In contrast, alcohol dependent patients persisted to the first established behavioral strategy. The mOFCdorsal striatum functional connectivity was impaired in the alcohol-dependent patients, but increased in correlation with the duration of abstinence.

Conclusion Our findings support that the disruption of the mOFC-striatal circuitry contribute to the maldaptive persistent behaviors in alcohol dependent patients.

Psychiatry Investig 2013;10:266-272

Key Words Alcohol, Medial orbitofrontal cortex, Dorsal striatum, Decision-making, fMRI.

\section{INTRODUCTION}

Addiction is characterized by persistent drug-seeking despite negative consequences and high frequency of relapse. There is growing body of evidence that addicts are less flexible to improve their performances and are prone to make perservative responses in cognitive tasks. ${ }^{1,2}$ Recently, it has been hypothesized that these maladaptive persistent behaviors of addicts reflect drug induced brain-changes that cause modifications in the orbitofrontal cortex (OFC).,4

The OFC plays a crucial role in adaptive flexible behavior through its unique pattern of connections with crucial sub-

Received: December 7, 2012 Revised: January 16, 2013

Accepted: January 16, 2013 Available online: September 16, 2013

$\triangle$ Correspondence: Young-Chul Jung, MD, PhD

Department of Psychiatry, Yonsei University College of Medicine, Severance Mental Health Hospital, 119 Gyeongchung-daero 1926beon-gil, Gwangju 464-100, Republic of Korea

Tel: +82-31-760-9405, Fax: +82-31-761-7582, E-mail: eugenejung@yuhs.ac

(a) This is an Open Access article distributed under the terms of the Creative Commons Attribution Non-Commercial License (http://creativecommons.org/licenses/by$\mathrm{nc} / 3.0$ ) which permits unrestricted non-commercial use, distribution, and reproduction in any medium, provided the original work is properly cited. cortical associative learning nodes, such as the striatum and the basalateral amygdala. ${ }^{3}$ The OFC and striatum are activated in response to high-incentive rewards ${ }^{5,6}$ and implicated in representing predicted future rewards. ${ }^{7}$ Individual differences in decision making performances are proposed to be related to the functional integrity of the striatal system, which can distinguish learners from non-learners during reinforcement learning. ${ }^{8}$ Thus, the disruption of the OFC-striatal interactions might account for the maladaptive decision making patterns that characterizes addiction. ${ }^{3}$

Previously, we developed a novel computerized decisionmaking paradigm and reported that the top-down control of the OFC over the striatum is a critical component underlying individual differences in behavioral responses. ${ }^{9}$ In the present study, we applied a similar paradigm in order to estimate the relationship between behavioral performance and the OFCstriatal circuit in patients with alcohol-dependence. We hypothesized that the OFC-striatal functional connectivity should be altered and thus the top-down cognitive control of the prefrontal cortex on behavior would be weakened in alcohol de- 
pendent patients.

\section{METHODS}

\section{Participants}

Twenty-one patients with alcohol dependence were recruited through the Alcohol Dependence Clinic at the Severance Mental Health Hospital, where they were receiving treatment. All patients were required to satisfy DSM-IV diagnostic criteria for alcohol dependence in the absence of past or present history of medical, neurological and other Axis I psychiatric illnesses. Comorbid anxious and depressive symptoms were not considered as exclusion criteria unless they fulfilled DSMIV diagnostic criteria for a major depressive episode. No patient had a past or present history of psychoactive drug abuse or dependence, except nicotine.

Twenty-four healthy control subjects were matched for age, sex, education and estimated intelligence quotient to the patients (Table 1). General intelligence was estimated using the Standard Progressive Matrices. ${ }^{10}$ This study was carried out

Table 1. Demographic characteristics

\begin{tabular}{lcccc}
\hline & Alcohol dependent group $(\mathrm{N}=21)$ & Healthy control group $(\mathrm{N}=24)$ & $\mathrm{t} / \chi^{2}$ & $\mathrm{p}$ \\
\hline Age (yrs) & $32.5 \pm 5.6$ & $30.4 \pm 3.2$ & 1.500 & 0.144 \\
Sex (male/female) & $12 / 9$ & $14 / 10$ & 0.007 & 1.000 \\
Education (yrs) & $15.2 \pm 2.3$ & $16.1 \pm 1.4$ & 1.468 & 0.152 \\
Estimated IQ* & $103.5 \pm 3.8$ & $105.5 \pm 4.0$ & 1.583 & 0.122 \\
Alcohol Dependence Scale ${ }^{\dagger}$ & $23.1 \pm 8.6$ & $1.2 \pm 1.9$ & 10.265 & $<0.001$ \\
\hline
\end{tabular}

*intelligence Quotient was estimated using the Standard Progressive Matrices (Raven JC, 1936), ${ }^{\dagger}$ Alcohol Dependence Scale (Horn et al. 1984)

Figure 1. A: Design of Odd-Even-Pass task. The visual stimuli were presented for $2500 \mathrm{~ms}$ and then the screen asked to choose between odd, even or pass within $1500 \mathrm{~ms}$. Each response was immediately followed by a feedback and inter-stimulus interval was jittered from $1000 \mathrm{~ms}$ to $6000 \mathrm{~ms}$. B and C: Behavioral performance. Pass responses (B); Reaction time (C).

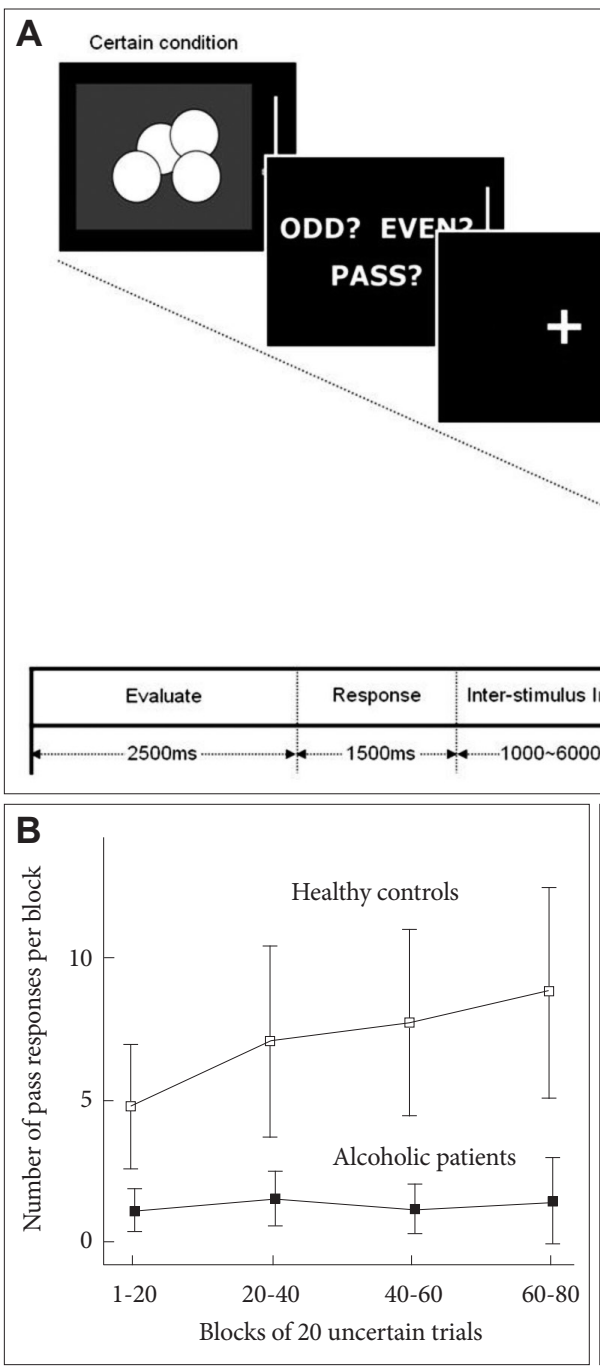

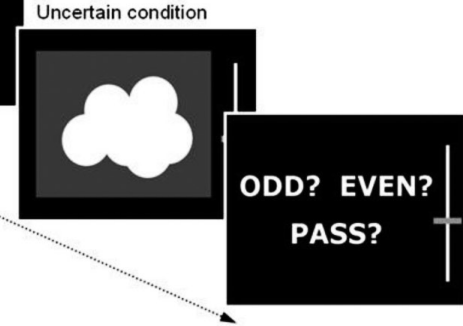
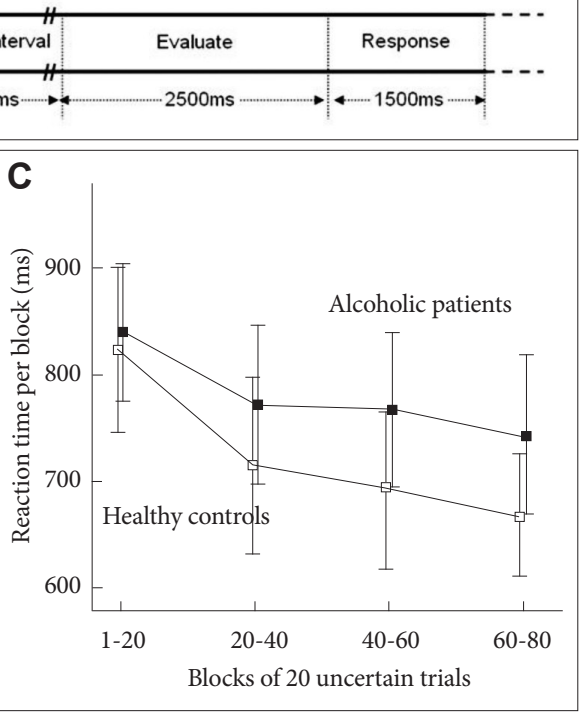
under the guidelines for the use of human participants established by the Institutional Review Board at Severance Mental Health Hospital, Yonsei University. Following a complete description of the scope of the study to all participants, written informed consent was obtained.

\section{Stimuli and experimental design}

Figures of coins were used as visual stimuli, which were presented through nonmagnetic goggles. The visual stimulus was presented for $2000 \mathrm{~ms}$ (evaluate phase) and then the participants were asked to guess whether the total number of coins was 'odd' or 'even' within $1500 \mathrm{~ms}$ (response phase) (Figure 1A). In addition to 'odd' and 'even', the participants were instructed that they could select a third option 'pass' in order to move on to the next trial without any gain or loss. Each response was immediately followed by a feedback (i.e., gain or loss one point).

The behavioral task was composed of two conditions: 1) a certain condition, in which the total number of the coins was two or three, so that the participants could easily guess the correct answer with little effort; 2) an uncertain condition, in which several coins were overlapped and the borders were blurred, making it impossible to exactly assess the correct number. The uncertain trials were designed to provoke a prediction error between the expected reward (at least a fifty-fifty chance) and the actual reward, which was predetermined by the computer to be fixed at $25 \%$ regardless of the participant's responses. We examined how long it took the participant to adapt a new behavioral strategy (i.e., make use of the alternative pass option). The task was composed of 40 certain trials and 80 uncertain trials, which were presented in a pseudorandomized order. The inter-stimulus interval was jittered between 1000 and $6000 \mathrm{~ms}$. We divided the task into four blocks (10 certain trials and 20 uncertain trials) in order to analyze the behavioral responses.

\section{Image acquisition and analysis}

Functional images were acquired on 1.5T GE scanner by using a gradient echo EPI sequence ( $\mathrm{TR}=2.5 \mathrm{~s}$ for the task session and TR=2 s for the resting session; TE=14.3 ms; flip angle $=90$; field of view $=240 \mathrm{~mm} ; 64 \times 64 \times 30$ matrix with $3.75 \times$ $3.75 \times 5 \mathrm{~mm}$ spatial resolution; 30 axial slices; and slice thickness $=5 \mathrm{~mm}$ ). A high resolution anatomical dataset was obtained for each subject by using a fast spoiled gradient echo sequence $(\mathrm{TR}=8.5 \mathrm{~s}, \mathrm{TE}=1.8 \mathrm{~ms}$, flip angle $=12$, field of view $=$ $240 \mathrm{~mm}, 256 \times 256 \times 256$ matrix with $0.94 \times 0.94 \times 1.5 \mathrm{~mm}$ spatial resolution, 116 axial slices, and slice thickness $=1.5 \mathrm{~mm}$ ).

The fMRI data were preprocessed using the Analysis of Functional Neuroimage program (AFNI) (Cox 1996). The first 7 time points in all the time series data were discarded. The rest of the data were performed slice timing correction, motion correction of all slices within a volume, and mean-based intensity normalization to convert the data from arbitrary intensity units to units of percent signal modulation. Further processing included spatial smoothing [Gaussian filter with $8 \mathrm{~mm}$ full-width at half-maximum (FWHM)]. Spatial normalization was performed to transform Talairach space using Montreal Neurological Institute (MNI) N27 template provided in AFNI (bilinear interpolation, spatial resolution: $2 \times 2 \times 2 \mathrm{~mm}$ ).

The response to each stimulus category (certain and uncertain) compared with the fixation baseline was calculated using multiple regression. All areas that showed a response to any stimulus type were included in the analysis. The impulse response function to each stimulus category was estimated with $2.5 \mathrm{sec}$ resolution using deconvolution. ${ }^{11}$ A separate regressor was used to model the response in each $2.5 \mathrm{sec}$ period in a 15 sec window following each stimulus presentation. With two stimulus types, this resulted in 14 regressors of interest (each consisting of a series of delta functions) resulting in an estimate of the response to a single stimulus of each type with no assumptions about the shape of the hemodynamic response. The response magnitude to each stimulus type was calculated by summing the beta-weights of the regressors from the 3 rd through the 6th second of the response (capturing the positive bloodoxygenation level dependent (BOLD) response but not any poststimulus undershoot). ${ }^{12}$ For each subject, the regression model provided a single estimate of the response to each stimulus type in each voxel. One-sample t-tests were performed in order to determine which brain regions showed increased activity for contrast uncertain $>$ certain.

\section{Regions of interest (ROI)}

We performed the analysis of covariance with the activities of conditions (uncertain >certain) and behavioral measures (total number of pass responses) as covariates in order to identify an OFC region as a seed for functional connectivity analysis. We used an uncorrected threshold of $\mathrm{p}<0.001$. The right medial OFC (mOFC; $x, y, z=8,40,-5$ ) demonstrated increased activities during the uncertain condition in contrast to the certain condition. We selected the right $\mathrm{mOFC}$ as the region of interest and used it as a seed region for functional connectivity analysis.

\section{Functional connectivity analysis}

For the connectivity analysis, the preprocessed fMRI data were temporally band-pass filtered $(0.01-0.08 \mathrm{~Hz})$ to reduce low frequency fluctuation of the signal in the BOLD for a functional connectivity analysis. ${ }^{13}$ The reference time series during the task session were extracted respectively by averaging time series from voxels in the subject-specific ROIs, which 
had been extracted from the ANCOVA analysis. The functional connectivity maps of the orbitofrontal seed (Supplementary Figures 1 and 2) were obtained by a correlation analysis being conducted with the reference time series and time series from the rest of the brain, which correlation coefficients were converted to $\mathrm{Z}$-values representing functional connectivity strength with the orbitofrontal seed using Fisher's $\mathrm{Z}$ transformation (two-tailed threshold of $\mathrm{p}<0.001$ ). ${ }^{14}$ Then, we defined a right dorsal striatum target from the functional connectivity map in order to calculate the mOFC-striatal functional connectivity strength in each participant.

\section{Behavioral analysis}

The number of pass responses per block (20 uncertain trials) was the dependent measure in the repeated measures of analysis of variance, with diagnosis as a between-group factor and block as a within-group factor. In addition, Pearson's correlation test was performed between the functional connectivity strength and behavioral responses (total number of pass decisions). Statistical analyses were conducted by using SPSS (SPSS Inc., Chicago, IL, USA) with two-tailed $\mathrm{p}<0.05$.

\section{RESULTS}

\section{Behavioral measures}

The number of pass responses increased as the uncertain trials repeated in the healthy control group. In contrast, the alcohol dependent group persisted to guess between odd and even. There were significant effects of group $(\mathrm{F}=13.366, \mathrm{df}=1$, $\mathrm{p}=0.001)$ and block $(\mathrm{F}=7.284, \mathrm{df}=3, \mathrm{p}<0.001)$ on the number of pass responses. In addition, there was a significant groupblock interaction $(\mathrm{F}=3.263, \mathrm{df}=3, \mathrm{p}=0.024)$ (Figure $1 \mathrm{~B})$. There was no significant difference between the two groups in the mean reaction time $(\mathrm{F}=3.234, \mathrm{df}=1, \mathrm{p}=0.079)$ (Figure $1 \mathrm{C})$.

\section{Functional connectivity map during the task session}

The alcohol dependent group and the healthy control group demonstrated distinct functional connectivity maps of the mOFC during the task. In the healthy control group, activities of the mOFC demonstrated significant correlations with activities of the amygdala, ventral striatum and dorsal striatum (Figure 2A). In contrast, there were significant correlations between the activities of the mOFC and the ventral striatum and dorsolateral prefrontal cortex in the alcohol dependent group (Figure 2B).

\section{Correlation between behavioral response and functional connectivity strength}

The mOFC-dorsal striatal functional connectivity strength significantly correlated with the number of pass responses in the healthy control group (Pearson Correlation $=0.420, \mathrm{p}=0.041$; Figure $2 \mathrm{C}$ ). In contrast, there was no correlation between the mOFC-dorsal striatum functional connectivity strength and pass responses in the alcohol dependent group (Pearson Correlation $=-0.083, \mathrm{p}=0.720$ ).
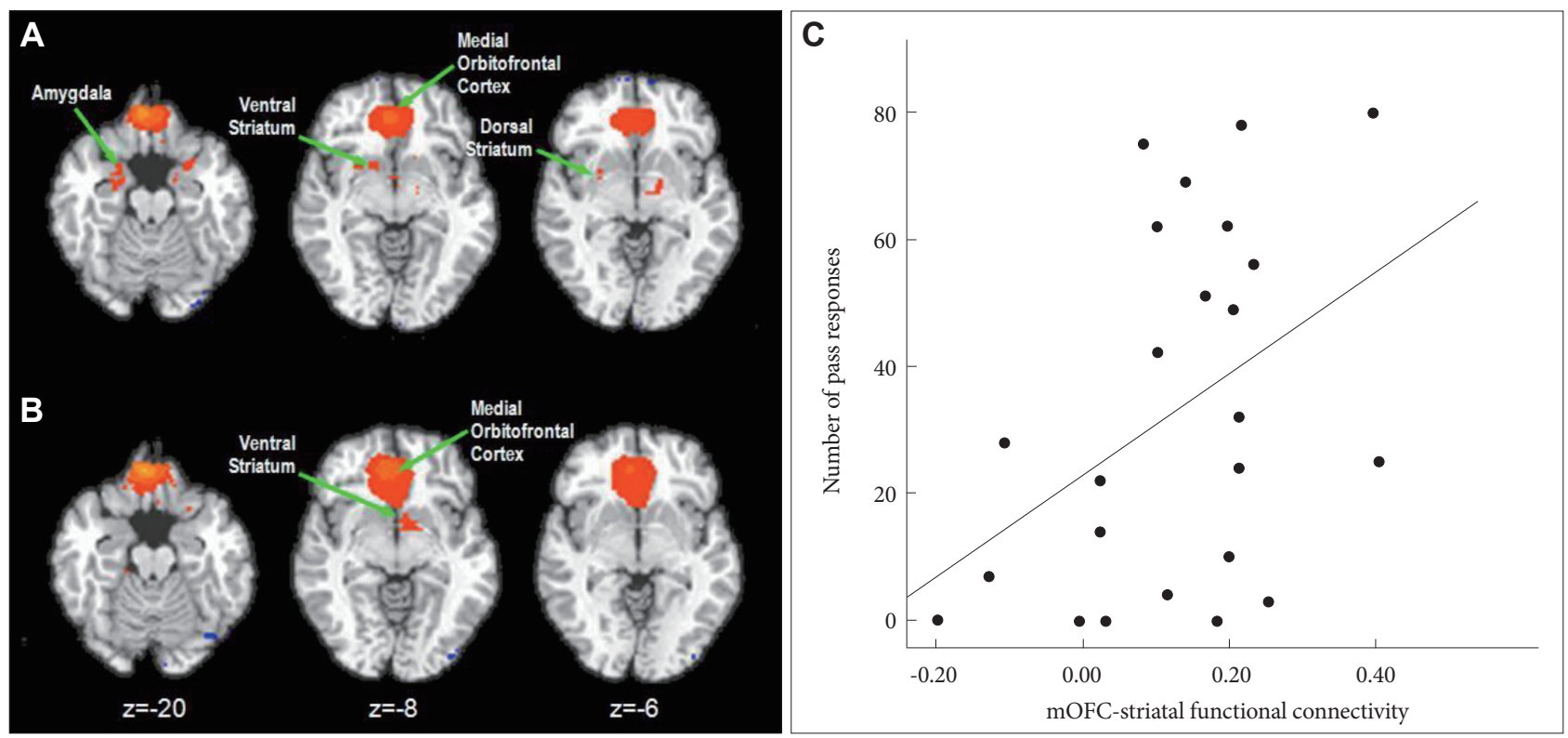

Figure 2. Functional connectivity of medial orbitofrontal cortex. The medial orbitofrontal cortex (mOFC) showed significant correlations with the amygdala, ventral striatum and dorsal striatum in the healthy control group (A). The mOFC showed significant correlations with only the ventral striatum in the alcohol dependent group (B). There was a significant correlation between the mOFC-dorsal striatum connectivity and the number of Pass responses within the healthy control group (C). 


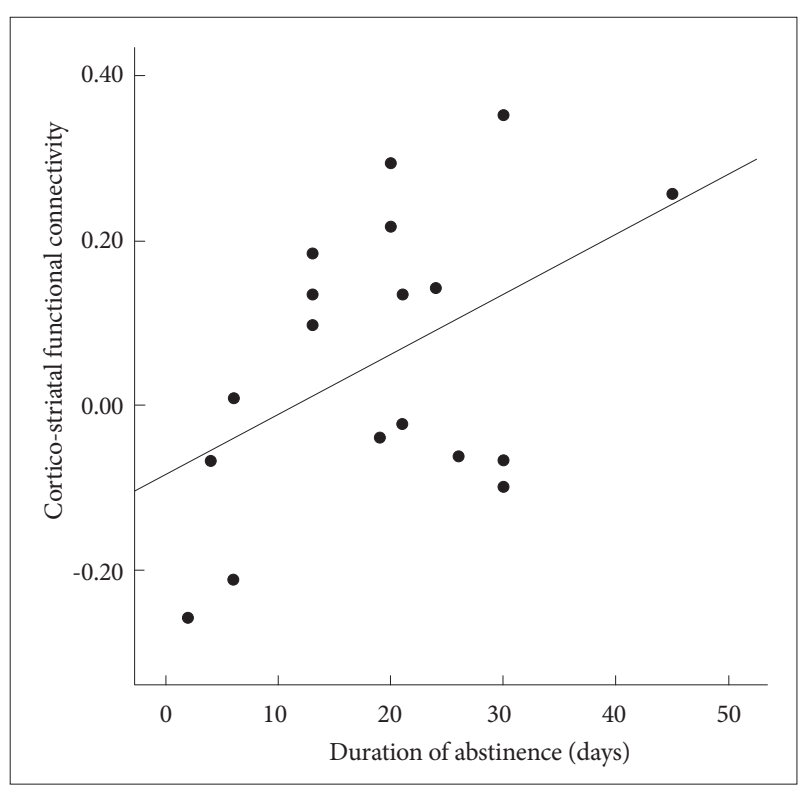

Figure 3. Correlation between connectivity strength and pass responses. In the alcohol dependent group, the medial orbitofrontal cortex-dorsal striatum connectivity correlated with the duration of abstinence.

\section{Correlation between duration of abstinence and functional connectivity strength}

The mOFC-dorsal striatal functional connectivity strength showed a correlation with the duration of abstinence in the alcohol dependent patients (Pearson Correlation $=0.470, \mathrm{p}=$ 0.049) (Figure 3).

\section{DISCUSSION}

In this study, we investigated the interconnected prefrontal and subcortical systems involved in adapting an alternative behavioral strategy. Our study revealed that the mOFC-dorsal striatum functional connectivity correlated with the tendency to adopt an alternative strategy in order to avoid high-risk uncertain situations. The mOFC-dorsal striatum functional connectivity was impaired in alcohol dependent subjects, who exhibited maladaptive persistent responses.

Persistence can be defined as the tendency to maintain a goal-directed behavior despite frustration, fatigue, and intermittent reinforcement. Persistence can be viewed as industrious, determination, and ambitiousness; however, when the contingencies change rapidly, persistence can be rigid and maladaptive. ${ }^{15}$ Persistence must be distinguished from perseveration, i.e., repetition of behaviors that are not obviously goal-directed, which is related to impairments in set-shifting and task switching. ${ }^{16}$ The mOFC-dorsal striatum functional connectivity was associated with switching strategies, whereas the mOFC-ventral striatum functional connectivity was related to persistent responses. ${ }^{9}$ Based on these findings, we speculate that the mOFC-striatal functional connectivity represents the top-down control of the prefrontal cortex over the striatum in action selection.

Decision making must consider both probability and the magnitude of each outcome. The dorsal striatum has been reported to encode information related to probability, whereas the ventral striatum has been consistently linked with processing information related to magnitude. ${ }^{17}$ The diverse functional connectivity among these components should contribute to the individual's differences in decision making. We speculate that the individuals with stronger functional synchrony between the mOFC and dorsal striatum were more guided by the probability of reward, which was predetermined at $25 \%$, and therefore adopted Pass as an alternative strategy to avoid the high-risk uncertain trials.

The striatum plays a crucial role in decision making, being involved not only in action selection but also in action evaluation. ${ }^{18}$ Our findings are consistent with the actor in actor-critic model of instrumental conditioning..$^{19,20}$ The actor-critic model proposes dissociable roles of the dorsal and ventral striatum: the dorsal striatum, as the actor, being critical for performance and the ventral striatum, as the critic, being important for learning. The OFC is proposed to moderate the interaction between the dorsal and ventral striatum. ${ }^{21}$ With regard to the actor-critic framework, substance-induced changes are proposed to interfere encoding in the ventral striatum, which result in abnormally strong initial learning and failure to change behavior. ${ }^{22}$ The dysfunction of the critic (ventral striatum) explains the increased impulsivity and sensitivity to reward magnitude in alcohol dependent subjects. ${ }^{23}$

The mOFC-ventral striatum functional connectivity was exhibited in both groups. It noteworthy, however, that the mOFCventral striatum functional connectivity correlated with behavioral performance only in the healthy control group, ${ }^{9}$ but not in alcohol dependent group, which likely indicates that the mOFC cognitive control over the striatal system was impaired in patients with alcohol dependence. The functional connectivity strength demonstrated correlation with the duration of abstinence in alcohol dependent patients. These findings are in line with the structural and metabolic neuroimaging studies that reported recovery of cortical grey matter and impaired frontal glucose metabolism in detoxified alcoholics. ${ }^{24,25}$

Another important finding is that functional connectivity between the mOFC and the amygdala was observed only in the healthy control group. The mOFC and basolateral amygdala are both implicated in learning representation of outcomes to guide behavior: the basolateral amygdala is critical for forming the representations, whereas the OFC is responsible for maintaining these representations and expressing them through behavior ${ }^{26}$ Accordingly, the maladaptive persistence 
of the alcoholics should be accounted by the interruption of the OFC-amygdala-striatum circuitry, rather than arising from discrete lesions in the striatum. On the other hand, the functional connectivity between the mOFC and the dorsolateral prefrontal cortex probably reflects the recruitment of additional brain regions in order to compensate for the impaired executive control in alcohol dependent subjects. ${ }^{27,28}$

The disruption of the orbitofronto-stiatal functional connectivity is not a specific finding of alcohol dependence, but also reported in other psychiatric diseases, such as obsessive compulsive disorder and depression. ${ }^{29,30}$ However, recent studies have reported that repeated episodes of detoxification from alcohol withdrawal are associated with decreased functional neural connectivity and increased vulnerability to emotional stress. ${ }^{31}$ Stronger functional connectivity between OFC and midbrain were found in abstainers compared with subsequent relapsers. ${ }^{32}$

There are some limitations to this study that preclude definitive conclusions. Our subjects were alcohol dependent patients still under treatment. Most of the patients were taking anti-craving agents and short-acting benzodiazepine medication for insomnia. The functional connectivity strength should have been influenced by confounding factors associated with the medication. In addition, four patients had been abstinent from alcohol drinking less than 10 days. It is hard to rule out the effect of withdrawal symptom on the behavioral performance as well as the functional connectivity strength in these patients.

In summary, the top-down cognitive control of the mOFC over the striatum appears to be a critical component of decision making and underlies the individual differences in addressing uncertain conditions. The impairment of mOFCdorsal striatum functional connectivity should contribute to the maladaptive perseverative responses in alcohol dependent subjects.

\section{Acknowledgments}

This study was supported by a grant from Yonsei University (6-2012-0182).

\section{REFERENCES}

1. Bechara A, Damasio H. Decision-making and addiction (part I): impaired activation of somatic states in substance dependent individuals when pondering decisions with negative future consequences. Neuropsychologia 2002;40:1675-1689.

2. Rogers RD, Everitt BJ, Baldacchino A, Blackshaw AJ, Swainson R, Wynne K, et al. Dissociable deficits in the decision making cognition of chronic amphetamine abuses, opiate abusers, patients with focal damage to prefrontal cortex and tryptophan depleted normal volunteers: Evidence for monoaminergic mechanisms. Neuropsychopharmacology 1999;20:322-339.

3. Schoenbaum G, Setlow B. Cocaine Makes action insensitive to outcomes but not extinction: implications for altered orbitofrontal-amygdalar function. Cereb Cortex 2004;15:1162-1169.
4. Everitt BJ, Wolf ME. Psychotomotor stimulant addiction: a neural systems perspective. J Neurosci 2002;22:3312-3320.

5. Delgado MR, Stenger VA, Fiez JA. Motivation-dependent responses in the human caudate nucleus. Cereb Cortex 2004;14:1022-1030.

6. Wilbertz G, van Elst LT, Delgado MR, Maier S, Feige B, Philipsen A, et al. Orbitofrontal reward sensitivity and impulsivity in adult attention deficit hyperactivity disorder. Neuroimage 2012;60:353-361.

7. O'Doherty JP. Reward representations and reward-related learning in the human brain: insights from neuroimaging findings. Curr Opin Neurobiol 2004; 14:769-776.

8. Schonberg T, Daw N, Joel D, O’Doherty JP. Reinforcement learning signals in the human striatum distinguish learners from nonlearners during reward-based decision making. J Neurosci 2007;27:12860-12867.

9. Jung YC, Ku J, Namkoong K, Lee W, Kim SI, Kim JJ. 2010. Human orbitofrontal-striatum functional connectivity modulates behavioral persistence. Neuroreport 2010;21:502-506.

10. O'Leary UM, Rusch KM, Guastello SJ. Estimating age-stratified WAISR IQS from scores on the Raven's Standard Progressive Matrices. J Clin Psychol 1991;47:277-284.

11. Glover GH. Deconvolution of impulse response in event-related BOLD fMRI. Neuroimage 1999;9:416-429.

12. Beauchamp MS, Lee KE, Haxby JV, Martin A. FMRI response to video and point-light displays of moving humans and manipulable objects. J Cogn Neurosci 2003;15:991-1001.

13. Biswal B, Yetkin FZ, Haughton VM, Hyde JS. Functional connectivity in the motor cortex of resting human brain using echo-planar MRI. Magn Reson Med 1995;24:537-541.

14. Zhou Y, Liang M, Tian L, Wang K, Hao Y, Liu H, et al. Functional disintegration in paranoid schizophrenia using resting-state fMRI. Schizophr Res 2007;97:194-205.

15. Svrakic DM, Cloninger CR. Personality Disorders. In: Sadock BJ, Sadock VA, Editors. Comprehensive Textbook of Psychiatry. Philadelphia: Lippincott Williams \& Wilkins, 2005, p.1724-29.

16. Evenden JL, Robbins TW. Increased response switching, perseveration and perseverative switching following d-amphetamine in the rat. Psychopharmacology (Berl) 1983;80:67-73.

17. Berns GS, Bell E. Striatal topography of probability and magnitude information for decisions under uncertainty. Neuroimage 2012;59:31663172 .

18. Kim H, Sul JH, Hun N, Lee D, Jung MW. Role of striatum in updating values of chosen acitons. J Neuroscience 2009;29:14701-14712.

19. Joel D, Niv Y, Ruppin E. Actor-critic models of basal ganglia: new anatomic and computational perspectives. Neural Netw 2002;15:535-547.

20. O’Doherty J, Dayan P, Schultz J, Deichmann R, Friston K, Dolan RJ. Dissociable roles of ventral and dorsal striatum in instrumental conditioning. Science 2004;304:452-454.

21. Atallah HE, Lopez-Paniagua D, Rudy JW, O’Reilly RC. Separate neural substrates for skill learning and performance in the ventral and dorsal striatum. Nat Neurosci 2007;10:126-131.

22. Takahashi Y, Schoenbaum G, Niv Y. Silencing the critics: understanding the effects of cocaine sensitization on dorsolateral and ventral striatum in the context of an actor/critic model. Front Neurosci 2008;2:86-99.

23. Roesch MR, Takahashi Y, Gugsa N, Bissonette GB, Schoenbaum G. Previous cocaine exposure makes rats hypersensitive to both delay and reward magnitude. J Neurosci 2007;27:245-250.

24. Pfefferbaum A, Sullivan EV, Mathalon DH, Shear PK, Rosenbloom MJ, Lim KO. Longitudinal changes in magnetic resonance imaging brain volumes in abstinent and relapsed alcoholics. Alcohol Clin Exp Res 1995;19:1177-1191.

25. Volkow ND, Wang GJ, Hitzemann R, Fowler JS, Overall JE, Burr G, et al. Recovery of brain glucose metabolism in detoxified alcoholics. Am J Psychiatry 1994;151:178-183.

26. Pickens CL, Saddoris MP, Setlow B, Gallagher M, Holland PC, Schoenbaum G. Different roles for orbitofrontal cortex and basolateral amygdala in a reinforcer devaluation task. J Neurosci 2003;23:11078-11084. 
27. Schulte T, Müller-Oehring EM, Sullivan EV, Pfefferbaum A. Synchrony of corticostriatal-midbrain activation enables normal inhibitory control and conflict processing in recovering alcoholic men. Biol Psychiatry 2012;71:269-278.

28. Chanraud S, Pitel AL, Müller-Oehring EM, Pfefferbaum A, Sullivan EV. Remapping the brain to compensate for impairment in recovering alcoholics. Cereb Cortex 2013;23:97-104.

29. Harrison BJ, Soriano-Mas C, Pujol J, Ortiz H, López-Solà M, Hernández-Ribas R, et al. Altered corticostriatal functional connectivity in obsessive-compulsive disorder. Arch Gen Psychiatry 2009;66:1189-1200.

30. Sakai Y, Narumoto J, Nishida S, Nakamae T, Yamada K, Nishimura T, et al. Corticostriatal functional connectivity in non-medicated patients with obsessive-compulsive disorder. Eur Psychiatry 2011;26:463-469.

31. O’Daly OG, Trick L, Scaife J, Marshall J, Ball D, Phillips ML, et al. Withdrawal-associated increases and decreases in functional neural connectivity associated with altered emotional regulation in alcoholism. Neuropsychopharmacology 2012;37:2267-2276.

32. Beck A, Wüstenberg T, Genauck A, Wrase J, Schlagenhauf F, Smolka $\mathrm{MN}$, et al. Effect of brain structure, brain function, and brain connectivity on relapse in alcohol-dependent patients. Arch Gen Psychiatry 2012; 69:842-852. 